\title{
Involving the elderly in the content development of a health enhancing tablet-based service
}

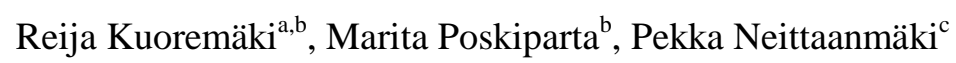 \\ ${ }^{a}$ Agora Center, ${ }^{b}$ Faculty of Sport and Health Sciences, Health Science, ${ }^{c}$ Faculty of Information Technology, Computer Science \\ P.O. Box 35, FI-40014 University of Jyväskylä, Finland
}

\begin{abstract}
The aim of the present study was to specify the needs of elderly for a health-enhancing tablet-based service. An interview study was performed with voluntary elderly $(\mathrm{N}=19$, female 15, male 4, aged 76.2 years (SD 8.3)). The attitudes towards improving health behaviours and technology utilisation were explored. The content illustrations were developed for the interviews, and evaluated by participants using a numerical scale (1 very poor - 5 very good) and verbal feedback. Content analysis was used in the analysis of qualitative data. Taking care of one's own well-being comprehensively was the most important incentive to maintain one's activity. Content illustrations were evaluated as high (average 2.9-4.4, current issues, memory training and exercise instructions as most useful). The diversity in wishes with respect to the contents of the service should be taken into account, and the customisation of the service will permit its use by different groups of end-users.
\end{abstract}

Keywords: elderly; health behaviour; mobile technology; tablet computer

\section{INTRODUCTION}

One of the most important challenges for societies is to encourage older persons to maintain their comprehensive functionality despite the normal physiological changes caused by aging [1]. Lifespans are increasing, and one target is to motivate the elderly to enhance their state of health so that more people can live autonomously longer. The need to improve the functionality of older people has been globally recognised [2]. Struggling with shrinking social life has been found to be common among the elderly when they are getting more impaired [3], so the active lifestyle should also be supported.

The digital divide and the importance of developing accessible services for the elderly have been recognised, and user involvement has been found to be a feasible method to contribute to the development of suitable services [4]. The acceptance of technology may be related to the state of health and the intention to use new technological services is strongly dependent on how useful and reliable the service is found by the user [5]. By involving the end-users in the development process of a service, emerging attitudes towards utilising technology and supporting health behaviour can be taken into account in the development of the content of the service.

Mobile technology has been used with promising results to improve the well-being of the elderly in several areas to promote functionality, e.g., physical activity, self-management of diseases and inquiries of health status [6-8]. Computer-based interventions have also been conducted with persons suffering mild cognitive impairment, and the results demonstrated that cognitive skills could be improved by memory training with well-designed technologically supported tasks [9]. Mobile technology enables the use of many features, e.g., text messaging [8], and different applications with media-rich content [7] in supporting health behaviour. The results of recent study demonstrate that health interventions via tablet computers are feasible in an elderly population; graphically demonstrated progress in training and the application was found to motivate individuals to perform exercise in patients not motivated to exercise before the use of the application [10]. Furthermore, adherence to such programs, e.g., in mobile rehabilitation, has been satisfactory, as was explored with the aged people in a previous study conducted at the University of Jyväskylä (Personal Mobile Space -project) [11].

The aim of the present study was to develop a new mobile optimised client-server web-service without complicated extra devices for inexperienced technology users. Considering the changing age structure of the population and its effects on the dependency ratio, the objective was specified not only to enhance health behaviours in the elderly but also to promote learning to use digital services in the preventive phase when they continue to live autonomously. The research question was "What motivates the elderly to comprehensively promote their health behaviour, and how a mobile technology based service should be designed to meet the needs of end-users".

\section{STUDY DESIGN}

The ideas for the content of the self-care service came from recent studies [7, 11] and available services, e.g., Sports Tracker and HeiaHeia (https://play.google.com/store), and some content was designed based on the ideas of researchers in the project. In total, twelve content illustrations were brainstormed, and the pictures were drawn by researchers (figure 1). First, useful information about services for the elderly in the city was designed to be provided via tablet. Themed information packages of, for example, the history of the city and various hobbies were considered to be interesting for end-users. Secondly, health behaviour support was considered comprehensively. To support physical activity, the idea of exercise instructions (figure 1) and self-monitoring of physical activity was proposed. Dietary guidelines were mentioned as one content illustration. To encourage older people to participate in social activities, information about current local events could be important. The social aspect was also designed to track physical activity as a social goal in the different sports. For cognitive training, memory games (figure 1) were thought to be interesting for elderly and easy to implement on mobile device. For mental well-being, the idea was proposed to include inquiries about mental tone to encourage individuals to self-monitor their own status.

The Finnish Innovation Fund Sitra, The Social and Healthcare Services City of 


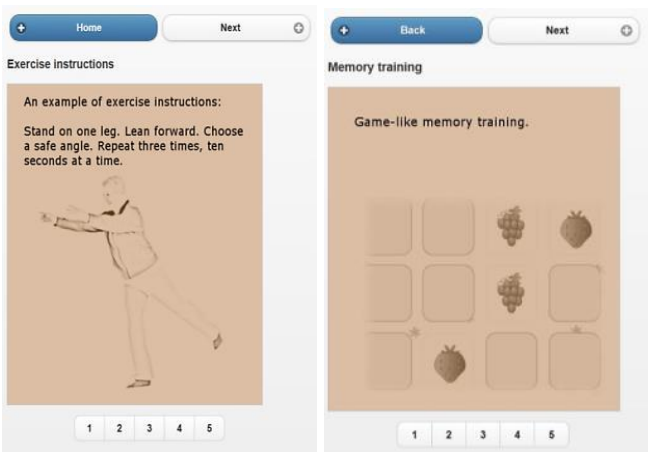

Figure 1. Examples of content illustrations of "exercise instructions" and "memory training". A draft of the picture and the main function of each idea were demonstrated

With respect to compliance, the game-like and entertainment aspects were brainstormed to be a part of the utility service to improve the attractiveness of the service. An option for self-monitoring physical activity was designed to be like a game; when one tracks some activity, a prize will be gained, such as a proverb or riddle. Additionally, allowing television and radio programs to be attained as part of the service was reasoned to be motivating. Motivational messages have been used in previous studies [7], so messages to encourage physical activity and reminders of the various events and health behaviours were considered. Many older people find the weather forecast useful, and a game-like option was brainstormed to make the one's own forecast and compare it with others'. The game-like solutions are worth exploring within groups of older people because, e.g., playing videogames has been noticed to be related to well-being in groups of older people [12]

\section{METHODS}

\section{Description of the Participants}

Based on the pre-study, the interviews were included with 19 older people (female (F) 15, male (M) 4), aged 76.2 (SD 8.3) years-old. The voluntary participants were recruited via employees of the social and healthcare services, City of Jyväskylä, Finland. The interviews were conducted in the autumn of 2012, from November to December, at the participants' homes.

Three of the participants were living in a group home and the rest autonomously (alone or with a spouse) or were receiving some public home care assistance. The participants were quite satisfied with their state of health, but some functional capacity limits were described; the most prevalent limit was the need for eyeglasses or hearing instruments. A lack of balance and mild memory impairment was also mentioned, but in general, the participants felt confident to deal with everyday tasks autonomously.

\section{Data collection}

The interview questions were semi-structured, and the interview proceeded on the basis of the responses by themes. The themes of interviews were as follows: supporting health behaviour, attitudes towards the use of technology and ideas for self-care service content. The duration of the interviews was $25-72$ minutes.

First, the participants were asked what type of things could encourage participation in sports or taking responsibility of own well-being. Some features that could be used to support health behaviour were discussed, such as cheering, reminders, instructions, game-like features, goals and external monitoring. Second, participants were asked about technology, including questions related to previous experiences, the use of information and communication technology, communicating or saving one's health data and sharing health data with relatives or healthcare personnel via technological services. Third, the content illustrations were presented to participants via a tablet device to make it easier to evaluate the need and the willingness to use different service components as well as to demonstrate the use of the tablet computer. The participants evaluated the content illustrations on a numerical scale (1 very poor - 5 very good) with the Mobile jQuery HTML-inquiry via a tablet computer. The pros and cons of the content illustrations were discussed as well.

\section{Analysis of the data}

In content analysis, the coding frame was created using the main themes of the interview. The concept-driven approach was used when categorising the data into the following major categories: "supporting health behaviour", "utilising technology" and "contents of the service". The data-driven sub-categories were formed to specify the meanings of the main categories [13]. Categorisation was performed carefully by the first author and was discussed in cooperation with the second author. The Atlas.ti 7.0 was used to code the data. The data were analysed to determine what motivates better health behaviours in the elderly and to determine how the opinions of the interview participants could be utilised in developing the Vetreeni service. The mean score for each evaluation of content illustration was calculated using Excel, and the suitability of tablet computer to answer inquiries was evaluated.

\section{RESULTS}

\section{Supporting health behaviour}

The participants' physical activities included everyday activity and some hobbies. Setting a goal was not deemed essential; taking care of one's own well-being, both mental and physical, was the most important incentive to participate in sports and in events. The different rehabilitation periods helped improve motivation, but the participants noted the difficult in maintaining motivation on one's own. Many of the participants thought that external monitoring of health behaviours could be helpful to maintain motivation; however, some participants did not like the idea of setting a goal or of someone looking after reaching the goal.

Different instructions were identified as important, but the participants expressed confusion in identifying the most important instructions for health behaviours because so many types of guidance are provided in magazines and television programs. Many of the participants recognised the importance 
of activities, and senior citizens clubs were especially popular because of the social aspect. Feeling safe was identified as essential role; if the participants' physical functionality were impaired, transportation services or support would be a prerequisite for safety.

\section{Utilising Technology}

The participants were not very familiar with technology use; some of the participants had experiences with home measurements of blood pressure or the use of step counters or medical device related to monitoring previously diagnosed disease symptoms. Several individuals used computers, but they were not familiar with tablet computer. Despite the fact they were not familiar with the touch screen, they felt quite confident about using it and were interested in learning. The participants were asked whether the service should be developed to work with the tablet computer or via television with an internet connection. Most participants thought that the tablet would be easier because of its portability and the ease in bringing up the service via the tablet device. The simplicity of not having extra devices was mentioned in favour of tablet computer.

The attitude towards saving health data to the national health database (Taltioni) was open-minded. Saving and sharing information with healthcare personnel and relatives was thought to be important because of the importance in understanding an individual's health state if he or she were not capable of taking care of medication or his or her health. Information security was mentioned several times. One of the concerns with respect to the development of new technologybased systems was how the elderly would be able to adapt to using services whose features are constantly changing.

\section{Results of the inquiry}

One of the participants did not want to answer the inquiries, so the content illustrations were evaluated by 18 participants. The results are presented in figure 2 . The most useful and interesting content illustrations were current events and services, memory training and exercise instructions, with mean score of $4.4,4.2$ and 4.2 , respectively (1 very poor -5 very good).

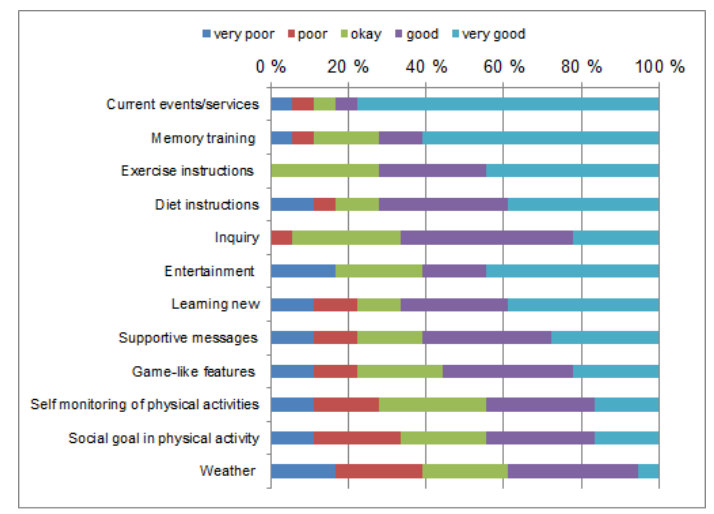

Figure 2. Results of the inquiry: the evaluation of each of the content illustrations ( $\mathrm{n}=18$; 1 very poor -5 very good).
The least interesting content illustrations were weather, social physical activity goals and self-monitoring of physical activity, with mean scores of 2.9, 3.2 and 3.2, respectively. The suitability of the tablet device to respond to the inquiries was evaluated as high (4.3).

\section{Verbal feedback on the contents}

A large number of verbal comments were gathered about each content illustration. The interviewees commented that information should be easily available. Some of the participants thought that a newspaper was adequate for information, but the content illustrations were generally evaluated as very useful. Memory training was important for the participants; the elderly participants stated the importance of rehearsing cognitive skills because of their decreasing abilities to remember things. The elderly participated in many hobbies related to memory exercise as well. Older people recognise the problems in functionality with ageing. These individuals know that participation in sport and physical activity is of increasing importance with age; if one chooses not to participate, functionality will suffer. Exercise instructions were evaluated as valuable and the major concern was that the exercises be safe. One of the main benefits of exercising was related to socialisation.

When describing the inquiry, the participants mentioned that the effects on mental well-being was important; the participants stated that it would be nice if someone sends inquiries so that they would feel that some people were interested in their feelings. With respect to diet instructions, the participants noted that many instructions are available in magazines and on television. Weight-controlling advice was considered particularly useful for those with functional limits to exercising. The supportive messages were evaluated as motivating by some participants; another did not want external cheering via the service.

Integrating entertainment content in the service was evaluated as useful; if one forgot to watch or listen to an interesting program via television or radio, the program could be easily watched via the tablet. Some of the participants already used the internet for this purpose. The educational aspects were also described as interesting by the participants. Some participants were interested in history, but there could be some prompts related to health issues also. It is possible that the game-like aspect was not employed in the optimal manner and may not be familiar to older people. Some of the participants found the games useful; some did not like the games at all. Self-monitoring of physical activities was not a very popular aspect of the service for everyone, but many thought that it might be useful to improve exercise motivation. The implementation of social physical activity goals was met with inconsistent feedback; for many of the participants, it was not easy to think metaphorically about the amounts of exercise. The participants suggested that weather be included in the service, but the option to predict the forecast did not receive much support from participants. 


\section{DISCUSSION}

Based on findings of the study, the elderly have openminded attitudes toward the use of technology to support health behaviours despite their limited experiences. Recent studies have proven that mobile technologies have been used to support the health behaviours of older people with good results $[6,7,10,14]$. User involvement has been used in the development of supportive applications, e.g., for people suffering mild dementia [15], and has proven to be a good means of gathering information about the content of the services in accordance with the wishes of older people.

The elderly participants found current issues, memory training and exercise instructions the most useful content to include in the Vetreeni service. Learning to use technology may also cause positive effects in social interactions (e.g. by receiving updates of current events) and decrease feeling loneliness [16]. The memory training was evaluated highly, and the contents of the tests should be diverse based on the wishes of participants and recent studies, e.g. memory tasks and mnemonics [17, 18]. Memory training is a promising method to improve memory skills, especially for persons suffering mild cognitive impairment [17]. Mobile technology has been found to improve mental wellness and self-efficacy; therefore, mobile technology-based interventions could have long-term effects [14].

Physical exercises are important to individual functionality [2], consistent with the opinions of older people. Inquiries and prompts about the use of the Vetreeni service were designed to be sent as a part of messaging systems to encourage participants to think about their healthy habits and the benefits of the tablet-service. Informational messages can improve engagement in health-related interventions, and active communication instead of passive information messages has also been found to be more interesting for participants [7], so the inquiries could be highly motivating. The feedback about physical exercise was very individual-specific; the participants were aware of the importance of safety when performing physical exercise, so the acceptance of technological service might be improved if the contents were developed with increased personalisation based on one's state of health [5].

Supporting well-being and functionality through a holistic approach should be considered in service development because many aspects of health are dependent on each other [1]. To prevent digital divide [2] and shrinking of the social life of the elderly [3], adequate services should be developed, and user involvement is critical in the developmental process. The next phase was to explore user experiences in a study with more participants. Based on these results, the development of the Vetreeni service will continue with superior knowledge and understanding of the wishes of the elderly.

\section{ACKNOWLEDGEMENT}

We thank participants of the study and colleagues working in the project, and the Taltioni cooperative of the possibility to integrate Vetreeni service into the Taltioni.

\section{REFERENCES}

[1] V. Gremeaux, M. Gayda, R. Lepers, P. Sosner, M. Juneau and A. Nigam, "Exercise and longevity," Maturitas, vol. 73, pp. 312-317, 12, 2012.

[2] ACSM and AHA, "Physical Activity and Public Health in Older Adults: Recommendation from the American College of Sports Medicine and the American Heart Association (ACSM/AHA)," Geriatr. Nurs., vol. 28, pp. 339-340, 0, 2007.

[3] T. Greenhalgh, J. Wherton, P. Sugarhood, S. Hinder, R. Procter and R. Stones, "What matters to older people with assisted living needs? A phenomenological analysis of the use and non-use of telehealth and telecare," Soc. Sci. Med., vol. 93, pp. 86-94, 9, 2013.

[4] D. Castilla, A. Garcia-Palacios, J. Bretón-López, I. Miralles, R. M. Baños, E. Etchemendy, L. Farfallini and C. Botella, "Process of design and usability evaluation of a telepsychology web and virtual reality system for the elderly: Butler," International Journal of HumanComputer Studies, vol. 71, pp. 350-362, 3, 2013.

[5] S. Sintonen and M. Immonen, "Telecare services for aging people: Assessment of critical factors influencing the adoption intention," Comput. Hum. Behav., vol. 29, pp. 1307-1317, 7, 2013.

[6] J. Joe and G. Demiris, "Older adults and mobile phones for health: A review," J. Biomed. Inform., vol. 46, pp. 947-954, 10, 2013.

[7] P. Klasnja and W. Pratt, "Healthcare in the pocket: Mapping the space of mobile-phone health interventions," J. Biomed. Inform., vol. 45, pp. 184-198, 2, 2012.

[8] B. H. Kim and K. Glanz, "Text Messaging to Motivate Walking in Older African Americans: A Randomized Controlled Trial," Am. J. Prev. Med., vol. 44, pp. 71-75, 1, 2013.

[9] C. Herrera, C. Chambon, B. F. Michel, V. Paban and B. Alescio-Lautier, "Positive effects of computer-based cognitive training in adults with mild cognitive impairment," Neuropsychologia, vol. 50, pp. 1871-1881, 7, 2012.

[10] P. Silveira, E. van het Reve, F. Daniel, F. Casati and E. D. de Bruin, "Motivating and assisting physical exercise in independently living older adults: A pilot study," Int. J. Med. Inf., vol. 82, pp. 325-334, 5, 2013.

[11] R. Kuoremäki, K. Ahde, A. Heinonen, J. Multanen and P. Neittaanmäki, "Feasibility of mobile health for rehabilitation - pilot study in finland," Proceedings of Med-e-Tel 2012: Global Telemedicine and eHealth updates: Knowledge Resources, Vol. 5. 2012.

[12] J. C. Allaire, A. C. McLaughlin, A. Trujillo, L. A. Whitlock, L. LaPorte and M. Gandy, "Successful aging through digital games: Socioemotional differences between older adult gamers and Nongamers," Comput. Hum. Behav., vol. 29, pp. 1302-1306, 7, 2013.

[13] M. Schreier, Qualitative Content Analysis in Practice. Thousand Oaks (Calif.): Sage Publications, 2012.

[14] V. Harrison, J. Proudfoot, P. P. Wee, G. Parker, D. H. Pavlovic and V. Manicavasagar, "Mobile mental health: Review of the emerging field and proof of concept study," Journal of Mental Health, vol. 20, pp. 509$524,2011$.

[15] M. Span, M. Hettinga, M. Vernooij-Dassen, J. Eefsting and C. Smits, "Involving people with dementia in the development of supportive IT applications: A systematic review," Ageing Research Reviews, vol. 12, pp. 535-551, 3, 2013.

[16] H. Blažun, K. Saranto and S. Rissanen, "Impact of computer training courses on reduction of loneliness of older people in Finland and Slovenia," Comput. Hum. Behav., vol. 28, pp. 1202-1212, 7, 2012.

[17] M. R. Olchik, J. Farina, N. Steibel, A. R. Teixeira and M. S. Yassuda, "Memory training (MT) in mild cognitive impairment (MCI) generates change in cognitive performance," Arch. Gerontol. Geriatr., vol. 56, pp. 442-447, 0, 2013.

[18] J. Reijnders, C. van Heugten and M. van Boxtel, "Cognitive interventions in healthy older adults and people with mild cognitive impairment: A systematic review," Ageing Research Reviews, vol. 12, pp. $263-275,1,2013$. 